\title{
Manfred Mai \\ Steuerungstheoretische Überlegungen über die veränderte Rolle des Staates bei technischen Infrastrukturen
}

Das Thema berührt drei Problemkreise:

- die ordnungspolitische Diskussion über die Rolle des Staates in der modernen Gesellschaft,

- die theoretische über die Steuerungsfähigkeit des Staates und

- die Steuerbarkeit gesellschaftlicher Teilsysteme, in dem Fall die Besonderheit technischer Infrastrukturen.

Diese drei Diskurse gilt es miteinander zu verbinden: Man kann den allgemeinen Diskurs über die Rolle des Staates nicht trennen von der Frage nach seiner besonderen Rolle in der Gestaltung und Regulierung technischer Infrastrukturen. Dennoch lassen sich etwa Erfahrungen mit dem Rückzug des Staates aus der Sozialverwaltung oder kommunalen Dienstleistungen nicht ohne weiteres übertragen auf die Privatisierung großer technischer Netzwerke. Deren Besonderheiten gilt es also gegenüber anderen Regulierungsbereichen herauszuarbeiten.

In allen Staaten - gerade auch in denen des ehemaligen Ostblocks - scheinen sich die Leitbilder der Privatisierung und Deregulierung (nicht nur technischer Infrastrukturen) durchgesetzt zu haben. Die Gründe dafür sind vielfältig: Sie reichen vom Scheitern planwirtschaftlicher Regime bis zur Sanierung öffentlicher Haushalte. In den letzten Jahren häufen sich die Fälle, in denen die Erwartungen an eine privatisierte Infrastruktur nicht erfüllt und Forderungen nach erhöhter öffentlicher Kontrolle lauter werden. Beispiele dafür sind u. a. die Unfälle der englischen Eisenbahn, der Zusammenbruch der Stromversorgung im Raum New York im Sommer 2002 oder die Unzufriedenheit mit der Deutschen Bahn und Telekom.

\section{Die Rolle des Staates in der modernen Gesellschaft}

Die Rolle des Staates in der modernen Gesellschaft wird in mehreren Diskursen und sozialwissenschaftlichen Teildisziplinen thematisiert. Zu den Gemeinsamkeiten aller Diskurse über die Rolle des Staates gehört die Erkenntnis, dass der moderne Staat gezwungen ist, seine Souveränität mit denen zu teilen, die die Adressaten seiner Politik sind und die in der Gesellschaft über Macht verfügen. Trotz ideologischer und wissenschaftstheoretischer Unterschiede ist das eine der Erkenntnisse, die alle sozialwissenschaftlichen »Lager« verbindet, obwohl die daraus zu ziehenden 
politischen Konsequenzen recht unterschiedlich sind. Was die einen ${ }^{1}$ als Verlust staatlicher Souveränität beklagen, sehen andere als zeitgemäße Form staatlichen Handelns ${ }^{2}$.

Es ist kein Zufall, dass konservative Autoren wie Ernst Forsthoff und Helmut Schelsky vornehmlich in der Technik und der mit ihr verbundenen Industrialisierung die Hauptursache für die Aushöhlung staatlicher Souveränität sehen. Liberale Autoren $^{3}$ gehen dagegen davon aus, dass der Staat seine Souveränität unter den Bedingungen einer differenzierten Gesellschaft mit mächtigen Akteuren nur dann erhalten und legitimieren kann, wenn er die gesellschaftlichen Akteure - Verbände, Unternehmen, NGO - in seine Politik einbindet, und zwar nicht nur unter dem Aspekt des effektiven Vollzugs, sondern auch unter dem der gemeinsamen Zieldefinition. Insofern deckt sich die politische Forderung nach Autonomie gesellschaftlicher Teilbereiche und Institutionen mit steuerungstheoretischen Einsichten ${ }^{4}$.

Eine Folge der wachsenden Einbindung gesellschaftlicher Akteure in die Aufgaben der Exekutive (z. B. bei der Vorbereitung, Evaluierung, Novellierung und beim Vollzug von Gesetzen und Verordnungen) ist ein steigendes Demokratiedefizit. Es wächst nicht nur mit der zunehmenden Intransparenz politischer Netzwerke sondern auch mit der Machtzunahme der Exekutive zu Lasten des Parlaments. Beispiele dafür sind die verschiedenen Kommissionen, Beiräte und »Runden Tische« der Bundesregierung ${ }^{5}$, die etwa die Arbeiten von Enquête-Kommissionen des Bundestages relativieren, wenn nicht gar aushebeln. Der »verhandelnde Staat « ${ }^{6}$ ist nämlich im wesentlichen die Regierung und Verwaltung. Dem Parlament verbleibt nur die punktuelle Kontrolle des Verfahrens und der Ergebnisse. Es verfügt strukturell und personell nicht über die Ressourcen, um auf die immer komplexer werdenden Politikfelder zu reagieren ${ }^{7}$. Diese Entwicklung ist nicht reversibel. Je mehr gesellschaftliche Bereiche es gibt, umso mehr Akteure und organisierte Interessen und damit

1 So zum Beispiel H. Schelsky, Auf der Suche nach der Wirklichkeit, Düsseldorf 1965, und E. Forsthoff, Der Staat der Industriegesellschaft, München 1971.

2 H. Schulze-Fielitz, »Staatsaufgabenentwicklung und Verfassung. Zur normativen Kraft der Verfassung für das Wachstum und die Begrenzung der Staatsaufgaben « in: D. Grimm (Hg.), Wachsende Staatsaufgaben - sinkende Stenerungsfähigkeit des Rechts, Baden-Baden 1990, S. 11- 47; F. W. Scharpf, "Die Handlungsfähigkeit des Staates am Ende des Zwanzigsten Jahrhunderts « in: Politische Vierteljabresschrift 1991, S. 621-634; R. Mayntz, »Triebkräfte der Technikentwicklung und die Rolle des Staates« in: G. Simonis / R. Martinsen / T. Saretzki (Hg.), Politik und Technik, PVS-Sonderheft 31, Wiesbaden 2001, S. 3-18.

3 U. von Alemann / R. G. Heinze, »Kooperativer Staat und Korporatismus. Dimensionen der Neo-Korporatismusdiskussion « in: U. von Alemann (Hg.), Neokorporatismus, Frankfurt/New York 1991, S. 43-61; F. Lehner, »Pluralistische Interessenvermittlung und staatliche Handlungsfähigkeit « in: U. von Alemann / E. Forndran (Hg.), Interessenvermittlung und Politik, Opladen 1983, S. 102-115.

4 G. Lehmbruch, »Der Beitrag der Korporatismusforschung zur Entwicklung der Steuerungstheorie« in: Politische Vierteljabresschrift 1996, S. 735-751.

5 R. G. Heinze, Die Berliner Räterepublik. Mehr Rat als Tat?, Opladen 2002.

6 R. Czada, Dimensionen der Verbandlungsdemokratie, Arbeitspapiere aus der FernUniversität Hagen, Polis 46/2000. 
um so mehr issues und Politikfelder entstehen, die für die Politik einen Gestaltungsbedarf erzeugen ${ }^{8}$.

Indem der Staat - seine Exekutive - mit Vertretern anderer Teilbereiche über Ziele und die Implementation von Maßnahmen verhandelt, erkennt er ihre Kompetenz und Machtposition an und festigt sie mit jeder weiteren Beteiligung9. Welche Einfluss- und Gestaltungsmöglichkeiten der Staat tatsächlich hat, hängt von den Konstellationen in dem jeweiligen Politikfeld ab. Das Spektrum staatlicher Gestaltungsmöglichkeiten reicht von aussichtslosen Verhandlungspositionen (Rücknahme negativer Standortentscheidungen internationaler Konzerne) bis zur dominierenden Rolle (Erteilung von Mobilfunk-Lizenzen).

Die Motive des Staates, mit Akteuren anderer Bereiche zu verhandeln, sind unterschiedlich:

- Gewinnung von Informationen über die Besonderheiten des zu regulierenden Bereichs,

- Erkennung möglicher Widerstände und Implementationsprobleme,

- Definition von gemeinsamen Interessen und Zielen,

- Erleichterung des Gesetzesvollzugs.

In der Praxis lassen sich diese Motive kaum voneinander trennen. In nahezu jedem Politikbereich haben sich wegen der offenkundigen Vorteile des Verhandelns Netzwerke gebildet, die weder für die Öffentlichkeit noch für das Parlament transparent sind. Es entsteht vielmehr der Eindruck eng verwobener Fachbruderschaften in den Expertengremien auf allen Ebenen von der EU-Kommission bis zur Kommunalpolitik.

Eine Zerschlagung derartiger Netzwerke im Namen der Basisdemokratie würde wenig bringen. Was an Demokratie gewonnen wird, geht an Effizienz verloren. Dieser Zielkonflikt - Effizienz kontra Demokratie - ist für den planenden Staat konstitutiv. Er ist nur zu rechtfertigen, wenn die Verfahren, in denen die staatlichen Bürokratien verhandeln, legitimiert sind: Legitimation durch Verfahren. Gerade im Bereich der Technik gibt es eine Vielzahl von Gremien, deren Verhandlungsergeb-

7 Die Diskussion um die Einführung einer parlamentarischen Technikbewertung hat u. a. gezeigt, dass staatliche Aufgaben, die sich nicht eindeutig einem bestimmten Politikfeld zuordnen lassen, immer Probleme haben, sich institutionell zu verfestigen. Die Folgen und Risiken großer technischer Systeme betreffen immer mehrere Politikfelder ebenso wie ihr Normalbetrieb. In der Praxis kann dies zu Konflikten zwischen dem federführenden (und politisch verantwortlichen Ressort) und anderen betroffenen Ministerien oder Behörden führen.

$8 \mathrm{Ob}$ damit auch eine Tendenz zur Verrechtlichung verbunden ist, ist letztlich eine politische Frage. Die Politik hat immer die Option, bestimmte gesellschaftliche Teilbereiche der Selbstregulierung zu überlassen. Es reicht, wenn der "Schatten der Hierarchie « für die Akteure erkennbar bleibt.

9 Das kann neue und alternative Akteure (etwa NGO), die in einem bestimmten Politikfeld entstanden sind, behindern, da sie die etablierten Akteure und Netzwerke, die sich gelegentlich den Vorwurf der Verfilzung gefallen lassen müssen, herausfordern. So beanspruchen z. B. in der Sozialpolitik Selbsthilfegruppen neben den etablierten Sozialverbänden Sitz und Stimme in den Gremien der Administration. 
nisse für die Technikregulierung von großer Bedeutung sind (z. B. im Arbeits- und Immissionsschutz sowie in der technischen Normung). Ohne die zahlreichen Expertennetzwerke wäre der Staat mangels Sachverstand und Kapazitäten mit der Regulierung überfordert ${ }^{10}$. Zur Verhandlungsdemokratie gibt es daher keine überzeugende Alternative.

Die Ausdifferenzierung gesellschaftlicher Teilsysteme wie Wirtschaft, Kultur, Religion, Wissenschaft usw. ist in demokratischen Staaten nicht mehr umkehrbar. Teilweise haben sich diese Bereiche in langjährigen Kämpfen emanzipiert - etwa die Kunst aus den Fängen der Kirche oder die Presse aus den Fängen des Staates -, teilweise wurde ihre Autonomie durch die Verfassung - und damit durch einen politischen $\mathrm{Akt}^{11}$ - garantiert. Grundlegend dafür sind die Ideen der Gewaltenteilung und die des checks and balances gesellschaftlicher Kräfte, wonach kein Teilsystem über das andere dominieren soll.

Je länger die Autonomie gesellschaftlicher Teilbereiche faktisch besteht - unabhängig davon, ob sie politisch gewährt oder erkämpft wurde -, um so mehr entfalten diese eine spezifische Rationalität. Diese Rationalität kann sich u. a. durch Geschäftsordnungen, professionelle Ethiken, Korpsgeist, Fachsprachen und andere Symbolsysteme verfestigen. Hinzu kommt, dass in allen ausdifferenzierten Bereichen neben einer dominanten Rationalität immer auch andere Rationalitäten bestehen, die sich organisationsintern als Zielkonflikte zeigen können. Steuerungstheoretisch ist relevant, dass alle gesellschaftlichen Institutionen durch die Verknüpfung mit mehreren Rationalitäten und Kontexten anschluss- und diskursfähig sind - und damit auch politisch gestaltbar. Die Politik kann z. B. einer Standesorganisation das Alleinvertretungsrecht (etwa in der Landwirtschaftspolitik den Bauernverbänden zu Gunsten von Verbraucherorganisationen) entziehen oder finanzielle Zuwendungen kürzen. Die Politik kann auch das Gewicht der dominierenden Rationalität gegenüber anderen verschieben (z. B. mehr Effizienz statt Sicherheit bei technischen Infrastrukturen). Gesellschaftliche Teilbereiche entwickeln zudem im Lauf der Zeit Institutionen und Interessen, die ein politisches Eigengewicht haben. So wird die Neugestaltung eines Politikfeldes allein durch das Interesse der betroffenen Mitarbeiter einer Behörde am Erhalt der Arbeitsplätze erschwert. Die planende Politik muss daher diesen Aspekt von vornherein mitbedenken.

Eine immer weiter fortschreitende Ausdifferenzierung der Gesellschaft in immer mehr verselbstständigte Politikfelder erhöht auch das Risiko, dass erfolgreiche Problemlösungen in einem Bereich für andere ein Probleminput sind: Die Privatisie-

10 Eine basisdemokratische Alternative bestünde in der Abstimmung über jeden einzelnen Grenzwert und Standard. Diese unpraktikable Alternative schließt nicht aus, dass bei besonders sensiblen Grenzwerten, etwa im Atomrecht, die Anforderungen an die demokratische Legitimation erhöht werden. Gegen die Auflösung bestehender policynetworks spricht auch, dass viele politische Lösungen nur in der Atmosphäre des gegenseitigen Vertrauens entstehen können. Wer eine Lösung verhindern will, braucht nur vertrauliche Inhalte an die Presse zu geben.

11 E. Bolsinger, »Autonomie des Rechts? Niklas Luhmanns soziologischer Rechtspositivismus - Eine kritische Rekonstruktion« in: Politische Vierteljabresschrift 2001, S. 3-29. 
rung öffentlicher Infrastrukturen löst zwar die Finanzprobleme einer Kommune, belastet aber den Arbeitsmarkt. Ab einer bestimmten Anzahl teilautonomer Bereiche ist es eine Frage staatlicher Kapazitäten, ob und wie diese Interdependenzen noch gesteuert werden können. Hinzu kommt, dass es immer Folgen geben wird, die nicht bereichsspezifisch als Problem erkannt und gelöst werden (können) und dann quasi selbstverständlich der Politik angelastet werden.

Von der Politik erwarten die Gesellschaft und die unmittelbar Betroffenen eine Lösung auch solcher bereichsspezifischer Probleme. Der Verweis auf die Unzuständigkeit des Staates - sei es für eine neue Tarifpolitik der Bahn oder für Mieter in privatisierten Wohnungsbaugesellschaften - wird vom Bürger kaum akzeptiert und mit Vertrauensverlusten bestraft. Insofern hat auch die oft kritisierte »symbolische Politik « für den Erhalt der Stabilität der Gesellschaft und des Systemvertrauens eine Bedeutung. Ein Politiker, der von vornherein seine Machtlosigkeit bei der Lösung konkreter Probleme erklärt, braucht bei Wahlen gar nicht erst anzutreten. Auch in aussichtslosen Situationen wird der Eindruck ernsthaften Bemühens - und sei es in Form inszenierter Kämpfe - honoriert ${ }^{12}$.

Dadurch entsteht für die Politik eine »Kompetenzfalle«: Die Regierung muss in jedem Fall den Eindruck vermitteln, als sei sie in der Lage, eine Lösung zu finden und durchzusetzen. Verspricht sie zuwenig, ist ihre Wiederwahl gefährdet. Wenn sie aber ihre Lösungskompetenz zu hoch ansetzt, besteht die Gefahr, dass sie an den selbst gesteckten Zielen (etwa die Halbierung der Arbeitslosigkeit oder die Sicherung des Rentenniveaus) scheitert und sogar richtige Schritte in Richtung einer Problemlösung als vergeblich erscheinen. Nicht immer gelingt es der politischen Kommunikation seitens der Regierung, die Verfehlung selbst gesetzter Ziele widrigen externen Umständen (Osterweiterung, Ölpreis usw.) zuzuschreiben - die Opposition und die Medien werden schon dafür sorgen, dass es wie Politikversagen aus$\operatorname{sieht}^{13}$.

Auch in der modernen Gesellschaft mit ihren Erosionstendenzen in fast allen Großorganisationen bleibt also der Staat der wichtigste Adressat für die Lösung von Problemen jedweder Art. Die großen gesellschaftlichen Teilbereiche haben sich trotz mehr oder weniger entwickelter Selbststeuerungskapazitäten als unfähig erwiesen, die Nebenfolgen selbst ihres eigenen Tuns zu beseitigen. Weder hat es die

12 Aus der Niederlage (etwa beim Kampf um den Erhalt von Strukturhilfen der EU für eine Region) kann der moderne Machiavellist immer noch einen Mythos bilden und sein Ansehen bei den Wählern stärken, während der ehrliche Politiker mit Einsicht in die systembedingten Zwänge (z. B. die Reform der europäischen Regionalförderung) politisch erledigt ist.

13 Den konstruktivistischen Charakter politischer Probleme und ihrer Lösungen zeigt Offe am Beispiel der Arbeitslosigkeit: »Man erkennt die gefundene Lösung daran, dass das Problem auf einmal nicht mehr da ist. (...) Die Preisfrage ist nur noch, ob und wie es uns gelingt, diesen Zustand in einer Weise auszugestalten, die sozial und politisch einigermaßen unschädlich bleibt « (C. Offe, »Vollbeschäftigung? Zur Kritik einer falsch gestellten Frage« in: K. Bentele / B. Reissert / R. Schettkat (Hg.), Die Reformfähigkeit von Industriegesellschaften. Fritz W. Scharpf zu seinem 60. Geburtstag, Frankfurt/New York 1995, S. 240). 
Wissenschaft geschafft, einen Ethikkodex zu entwickeln, der einen akzeptablen Umgang mit bestimmten Gentechnologien ermöglicht, noch die Wirtschaft, die Ungleichheit der Güterverteilung in den Griff zu bekommen. Solange es bereichsspezifische Nebenfolgen gibt, die diese Bereiche selbst nicht lösen können, wird es Aufgabe des Staates sein, entweder sich der Lösung dieser Probleme direkt anzunehmen oder andere Akteure zu motivieren, diese Probleme zu lösen. Letzteres ist kein Beleg für staatlichen Souveränitätsverlust, sondern für problemangemessenes Handeln.

Es ist nicht zuletzt Aufgabe der Politik, überhaupt zu definieren, was als zu lösendes Problem gilt und was nicht. Ob die Bekämpfung bestimmter Hunderassen dringlicher sein kann als die der Armut ist eine politische Frage. Nur die Politik ist in der Lage, übergreifende Probleme zu erkennen und sie mit Hilfe anderer Teilbereiche zu lösen. Dem widerspricht nicht, dass auch politisches Handeln für die Wirtschaft oder die Wissenschaft zu einem Problem werden kann. Wenn z. B. aus politischen Gründen Beschlüsse gefasst werden (z. B. die Öffnung der Arbeitsmärkte im Rahmen der EU-Osterweiterung), kann das für bestimmte Wirtschaftsbranchen erhebliche Folgen haben (etwa die Zunahme illegaler Beschäftigung und Schwarzarbeit). Diese werden erst dann wieder zu einem politischen Problem, wenn es der Wirtschaft gelingt, die zunächst nur für sie auftretenden Folgen als gesellschaftliches Problem zu politisieren (z. B. als Aushöhlung sozialer Standards). Erst dann kann wiederum die Politik eine Lösung (Übergangsfristen oder Ausnahmeregelungen) erreichen.

In den verschiedenen politischen Ideologien ist die Rolle des Staates ebenfalls wie in den sozialwissenschaftlichen Diskursen - thematisiert. Auf der einen Seite gibt es die auf Thomas Hobbes zurückgehende Tradition des auf vertraglichen Regelungen beruhenden und über den konfligierenden Einzelinteressen stehenden Staates. Auf der anderen Seite steht die von John Locke entwickelte Idee eines Staates, der die Rechte des Individuums (vor allem seine Eigentumsrechte) gegen staatliche Eingriffe sichert ${ }^{14}$. Beide Denktraditionen spielen auch in der heutigen Politik und in den steuerungstheoretischen Diskursen eine Rolle.

So z. B. in der Frage der Verwaltungsreform. In den verschiedenen Leitbildern der Verwaltungsreform ist immer ein Teil des Staatsverständnisses zu finden, wie es diese beiden Klassiker (und andere) entwickelt haben. Wer den Bürger mit seinen Rechten an der freien Entfaltung in den Mittelpunkt stellt, wird die öffentliche Verwaltung auf ein Minimum beschränken wollen und jede überflüssige Bürokratie der Gängelung verdächtigen. Wer dagegen die Bürger in sozialstaatlicher Tradition vor den Folgen der Industrialisierung schützen will ${ }^{15}$, kommt zu einem anderen Leitbild der Verwaltung. Ein gemeinsamer Nenner aller Leitbilder zur Reform der öffentlichen Verwaltung ist die Forderung nach Verschlankung sowie erhöhter Transparenz, Effizienz und Bürgernähe: Der Bürger als »Kunde«, das Verwaltungshandeln als »Produkt « und die Behörde als »Dienstleister ${ }^{16}$. 
Der Staat zieht sich nicht zurück - das konkrete Handeln und Kooperieren seiner Regierung und Verwaltung wandeln jedoch seine Erscheinungsform: Aus dem hohheitlichen »Vater Staat « wird zunehmend der verhandelnde »Partner Staat «. Dies erfordert seitens der staatlichen Akteure vor allem ein anderes Selbstverständnis als das dominierende des Verwaltungsrechts. Der überwiegend mit Koordination, Verhandlung und Mediation befasste Staat braucht andere Instrumente als die des klassischen Verwaltungsrechts. Er braucht vor allem die Kompetenz, die unterschiedlichen Optionen technischer Infrastrukturen und ihre Folgen zu erkennen und mit den verschiedenen organisierten Interessen umzugehen, wenn er sich nicht von der »vereinten Dynamik von Globalisierung, Liberalisierung und Digitalisierung (...) »zum Zaungast « degradieren lassen will ${ }^{17}$.

Die veränderte Form des Staates erfordert auch von seiten der Partner entsprechende Änderungen. Nicht alle gesellschaftlichen Bereiche haben sich gleichermaßen emanzipiert und treten gegenüber dem Staat als selbstbewusste und autonome Akteure auf. Was für die Bereiche Wirtschaft und Wissenschaft gilt, muss nicht in den Bereichen des Sozialen und der Kultur gelten. Für großtechnische Systeme kann jedoch davon ausgegangen werden, dass deren Akteure dem Staat selbstbewusst gegenüber treten.

\section{Eigensinn und Rationalität technischer Infrastrukturen}

Bei der Frage nach der Steuerbarkeit technischer Infrastrukturen spielt die Besonderheit dieses Steuerungsobjekts eine Rolle. Was haben technische Infrastrukturen gemeinsam und was unterscheidet sie von anderen Steuerungsobjekten? Technischen Infrastrukturen und Netzwerken ist gemeinsam, dass in ihrem Mittelpunkt eine Technologie steht, die in einen organisatorischen Kontext eingebettet ist und der Erfüllung bestimmter Aufgaben dient. Typische Beispiele dafür sind: die Eisenbahn, das Telefon, die Wasser- und Energieversorgung. Die besondere Bedeutung technischer Infrastrukturen für die Politik ergibt sich aus ihrer zentralen Rolle für die Sicherung elementarer Grundfunktionen für die Gesellschaft ${ }^{18}$. Auch nur ein zeitweiliger oder regional beschränkter Ausfall eines dieser Netze hat in der Regel große Folgen für fast alle anderen gesellschaftlichen Bereiche. Weil technische Infrastrukturen für die Entwicklung eines Landes ebenfalls eine zentrale Rolle spielten und spielen, wurden sie zumindest in Europa zunächst fast nur unter staatlicher Aufsicht geführt ${ }^{19}$. Auch heute zeigt sich in vielen Entwicklungsländern, dass ohne eine zuverlässige Infrastruktur in den Bereichen Kommunikation, Verkehr und Energie fast alles andere vergeblich ist. Infrastrukturen sind die Achillesfersen jeder

16 W. Jann, »Politik und Verwaltung im funktionalen Staat« in: W. Jann / K. König / Ch. Landfried / P. Wordelmann (Hg.), Politik und Verwaltung auf dem Weg in die transindustrielle Gesellschaft. Carl Böbret zum 65. Geburtstag, Baden-Baden 1998, S. 253-280.

17 H. Willke, »Informationstechnische Vernetzung als Infrastrukturaufgabe - Welche Rolle spielt die Politik? « in: R. Werle / Ch. Lang (Hg.), Modell Internet? Entwicklungsperspektiven neuer Kommunikationsnetze, Frankfurt/New York 1997, S. 128. 
Gesellschaft und deshalb bevorzugte Schutzobjekte - nicht nur gegen terroristische Angriffe, sondern vor allem auch gegen Naturkatastrophen und technische Ausfälle.

Vor dem Hintergrund ihrer geschichtlichen Entwicklung wird verständlich, warum ihre Privatisierung in einigen Ländern nur zögernd in Angriff genommen wird. Hinzu kommt ein anderes Trägheitsmoment. Jede Infrastruktur hat im Laufe der Zeit einen beachtlichen Verwaltungsapparat aufgebaut, der alle Verwaltungsebenen vom Ministerium bis zur lokalen Behörde umfasst. Nur so schienen die Sicherheit und Qualität technischer Infrastrukturen gewährleistet. Unabhängig von der spezifischen Rationalität einer technischen Infrastruktur liefert ihr organisatorischer Mantel Ansatzpunkte für die politische Gestaltung dieser Infrastruktur. So kann die Politik durch Zuweisung oder Entzug finanzieller Ressourcen zum Aus- oder Abbau der Infrastruktur beitragen. Sie kann weiterhin qua Gesetz die Infrastruktur dezentralisieren (oder eine dezentrale Infrastruktur zentralisieren) oder Leistungsparameter definieren. Nicht zuletzt kann durch die politische Besetzung von Leitungsstellen ein Umbau der Infrastruktur in die gewünschte Richtung gelenkt werden (z. B. wenn es gilt, eine Infrastruktur zu privatisieren).

Dennoch haben diese akteurzentrierten Maßnahmen eine Grenze. Anders als beim Umbau einer Krankenkasse zeigt sich bei technischen Infrastrukturen ihre Prägung durch das Artefakt. Eine einmal implementierte Infrastruktur lässt sich nicht ohne weiteres durch eine andere Option ersetzen. Die Entscheidung für eine bestimmte Struktur der Energieversorgung und des Schienenverkehrs z. B. bedeutet eine Festlegung für mehrere Jahrzehnte. Die Festlegungen z. B. des Energiewirtschaftgesetzes aus den 1930er Jahren ${ }^{20}$ oder die Verkabelungspolitik aus den 1970er Jahren sind noch heute für die Politik relevant. Alternative Optionen - z. B. dezentrale Kleinkraftwerke statt zentraler Großkraftwerke - sind nur langsam und dann nur unter hohen Kosten möglich, aber sie sind zumindest denkbar. Einmal getroffene Entscheidungen für eine bestimmte technische Infrastruktur bedeuten für Generationen eine Pfadabhängigkeit für die weitere Entwicklung dieser Infrastruktur. Für die Politik bedeutet dies für eine lange Zeit eine Einschränkung möglicher Optionen. Aus diesem Grund gibt es unterschiedliche nationale Traditionen (»Pfade«)

18 Zur Definition »großer technischer Systeme«, die hier mehr oder weniger synonym mit technischen Infrastrukturen gleichgesetzt werden, vgl. R. Mayntz, »Große technische Systeme und ihre gesellschaftstheoretische Bedeutung « in: Kölner Zeitschrift für Soziologie und Sozialpsychologie 1993, S 97-108, sowie P. Weingart, " Großtechnische Systemer - ein Paradigma der Verknüpfung von Technikentwicklung und sozialem Wandel? « in: P. Weingart (Hg.), Technik als sozialer Prozeß, Frankfurt a. M. 1989, S. 174-196. Streng genommen sind große technische Systeme immer auch technische Infrastrukturen, aber nicht jede Infrastruktur ist ein großes technisches System. Politische Relevanz dürfte weniger eine gebrochene Wasserleitung haben als eine Störung der Stromversorgung.

19 Vgl. dazu die Fallstudie über das Telefon als Beispiel eines großen technischen Systems von F. Thomas, Telefonieren in Deutschland, Frankfurt/New York 1995.

$20 \mathrm{H}$. D. Hellige, »Entstehungsbedingungen und energietechnische Langzeitwirkungen des Energiewirtschaftgesetzes von 1935 in: Technikgeschichte Bd. 1986, S. 123-155. 
mit verschiedenen Modellen konkreter Infrastrukturen. Die Übertragbarkeit anderer Modelle (»best-practice «) scheitert in der Regel an der unterschiedlichen Einbettung der Infrastrukturen in das jeweilige nationale Gesellschaftssystem.

Die besonderen Steuerungsprobleme technischer Infrastrukturen haben noch andere Ursachen. Technische Netzwerke haben in der Regel einen hohen Investitionsund Wartungsbedarf und erschweren allein deshalb eine andere Option. Jedes große technische System hat seine »Restlaufzeiten «, deren Dauer politisch verhandelbar ist (Restlaufzeit gegen Rechtssicherheit). Die einmal getätigten Investitionen müssen sich nicht nur für die Industrie, sondern auch für die Politik »rechnen«.

Wegen der Langfristigkeit der Planung und Vielzahl von Einzelschritten (Teilgenehmigungen) beim Aufbau einer technischen Infrastruktur ist eine kontinuierliche politische Kontrolle und Steuerung erschwert. Die Entscheidung für den Einstieg in eine bestimmte Infrastruktur trifft in der Regel eine andere Regierung auf der Basis einer anderen Mehrheit und anderer Prioritäten als die Entscheidung für die weitere Nutzung dieser Infrastruktur. Was zu einem bestimmten Zeitpunkt als sinnvoll erscheint - etwa der Einstieg in die bemannte Raumfahrt -, kann sich später als teurer Luxus erweisen, von dem man sich wegen der langfristig abgeschlossenen Verträge mit internationalen Partnern nur schwer trennen kann. Die Verträge müssen langfristig abgeschlossen sein, weil sich andererseits keine Planungssicherheit für das Projekt herstellen lässt. Kurskorrekturen aufgrund der Änderung gesellschaftlicher Konstellationen (Wechsel politischer Mehrheiten, Bürgerproteste u. ̈̈.) lassen sich nur mit erheblichen Kosten (es drohen z. B. Schadensersatzklagen beteiligter Unternehmen oder bei internationalen Projekten Prestigeverlust) vornehmen.

Eine Lösung dieses Problems der ungleichzeitigen Entwicklung von technischer Infrastruktur und politischen Prioritäten wäre eine kontinuierliche Technikbewertung, die in erster Linie beim Parlament anzusiedeln wäre. Nur so ist sichergestellt, dass regierungsunabhängige Informationen und Bewertungen als Entscheidungsgrundlage über große technische Systeme zur Verfügung stehen ${ }^{21}$.

Technische Artefakte können ihren Gebrauch strukturieren - oder allgemeiner: Ihre Integration in soziotechnische oder Mensch-Maschine-Systeme determiniert bis zu einem gewissen Grad soziales Handeln ${ }^{22}$. Zwar hat der Abschied vom Technikdeterminismus ${ }^{23}$, wie ihn noch Schelsky in seinen Technokratiethesen vertreten hat, den Blick für organisatorische und damit auch politische Gestaltungsoptionen geöffnet. Programmatischer Ausdruck dafür waren die Programme »Humanisierung des Arbeitslebens $(\mathrm{HdA})$ « des damaligen Bundesforschungsministers und »Mensch und Technik - Sozialverträgliche Technikgestaltung (SoTech)« in NRW. Dennoch gibt es weder einen Organisations- noch Wertdeterminismus, bei dem sich technische Systeme als beliebig gestaltbar erweisen. Die relative Gestaltungsresis-

21 M. Mai, Technikbewertung in Politik und Wirtschaft, Baden-Baden 2001, S. 80.

22 G. Ropohl, Technologische Aufklärung, Frankfurt a. M. 1991, S. 183.

23 B. Lutz, »Das Ende des Technikdeterminismus und die Folgen - Soziologische Technikforschung vor neuen Aufgaben und Problemen« in: B. Lutz (Hg.), Technik und sozialer Wandel, Frankfurt a. M. 1987, S. 34-51. 
tenz einmal institutionalisierter technischer Systeme ergibt sich aus ihrer Vernetzung mit anderen sozialen Systemen, aus Kostengründen und auch aus physikalischen Grenzen. Es gibt deshalb z. B. nur eine begrenzte Zahl terrestrischer Sendefrequenzen, die die Medienpolitik zwingt, eine Auswahl zu treffen. Erst jetzt, wo im Zuge der Digitalisierung der Medien diese Grenze wegfällt, eröffnen sich auch für die Politik ganz andere Spielräume ${ }^{24}$. Ähnliches gilt für die Grenzen des Schiene-Rad-Systems oder die des Luftverkehrs.

Die konkrete Gestalt eines technischen Systems ist immer ein Kompromiss aus dem technisch Möglichen, dem wirtschaftlich Vertretbaren, dem gesellschaftlich Erwünschten und dem politisch Durchsetzbaren. Alle diese Faktoren können sich u. U. schnell ändern und die weitere Gestaltung eines technischen Systems ändern: Veränderungen auf Teilmärkten können die Wirtschaftlichkeit ebenso in Frage stellen wie die Veränderung politischer Prioritäten oder das Auftauchen neuer Innovationen.

Hinzu kommt eine spezifische Rationalität der Technikgenese. Die Entwicklung einzelner Technologien zielt immer auf eine Verbesserung definierter Parameter, sei es die Speicherdichte bei Chips, der Wirkungsgrad bei Kraftwerken oder der Komprimierungsgrad von digitalen Übertragungsmedien. Weil dieser Prozess quasi immanent nach den Regeln der jeweiligen Ingenieurwissenschaft abläuft, entsteht ein ständiger Innovationsdruck. Technische Infrastrukturen bedürfen daher stets einer technisch-organisatorischen Erneuerung, wenn sie noch dem »Stand der Technik « entsprechen wollen. Was in den 1980er Jahren als der neueste Stand der Kabeltechnik galt, ist heute ein Wachstumshindernis für die Multimediabranche, die Übertragungsraten braucht, die frühere Techniker für unmöglich hielten. Dieser permanente Innovationsdruck von seiten der Technik erfordert von der Politik ständig Entscheidungen darüber, ob sie die vorhandenen Netze modernisiert und in welcher Form sie sie weiterführen will. Dieser Druck wird dadurch verstärkt, dass sich jede Regierung in einem internationalen Standortwettbewerb um die fähigsten Infrastrukturen befindet.

\section{Funktionale Differenzierung oder intersystemische Penetration?}

Für die Frage nach der Steuerbarkeit technischer Infrastrukturen ist das dabei zugrundegelegte Gesellschaftsmodell entscheidend. Wer von einer funktional differenzierten Gesellschaft mit dem politischen System als eines von mehreren einander gleichrangigen Subsystemen ausgeht ${ }^{25}$, muss zu anderen Erkenntnissen kommen als wer weiterhin vom Primat der Politik ausgeht. Die Anerkennung der Politik als

24 M. Mai, »Strategien zur Sicherung der Rundfunkfreiheit. Die Rolle der Technik in der Massenkommunikation « in: R. Martinsen / G. Simonis (Hg.), Demokratie und Technik - (k)eine Wablverwandtschaft?, Opladen 2000, S. 109.

25 H. Willke, Ironie des Staates. Grundlinien einer Staatstheorie polyzentrischer Gesellschaft, Frankfurt a. M. 1992, S. 212. 
nach wie vor wichtigsten Bereich der Gesellschaft bestreitet nicht die relative Autonomie anderer Teilbereiche wie Wirtschaft, Wissenschaft und Kunst, die auf einem politischen Akt beruht. Diese Grundannahme ist etwas anderes als die Annahme emergenter Prozesse (»invisible hands«) ohne identifizierbaren Akteur.

Es ist fraglich, ob die moderne Gesellschaft überwiegend funktional differenziert ist oder ob es sich dabei nur um einen Idealtypus handelt, der allenfalls in einigen wenigen Modernitätsinseln unserer Gesellschaft aufscheint. In einer Gesellschaft, in der die Schattenwirtschaft, Migration, organisierte Kriminalität sowie regionale und ethnische Konflikte immer wieder auf den vorderen Plätzen der politischen Agenda auftauchen, sollte man besser von anderen Differenzierungsformen sprechen, die mitnichten historisch überwunden sind. Selbst hochentwickelte Organisationen wie Universitäten, Unternehmen und Verwaltungen sind alles andere als funktional differenziert, sondern durch ein Nebeneinander von formellen und informellen Strukturen gekennzeichnet, in denen nicht zuletzt der Rolle von Persönlichkeiten oder Eliten ein gewisses Gewicht zukommt. Der richtige Mann oder die richtige Frau an der Spitze eines Verbandes oder einer Forschungseinrichtung kann Gestaltungschancen erkennen oder Änderungen zum richtigen Zeitpunkt veranlassen - unabhängig davon, ob diese Organisation einem spezifischen Code folgt. Es wäre dann immer noch zu klären, worin dieser spezifische Code eigentlich besteht.

Großtechnische Netzwerke sind in mehrere Kontexte eingebunden und werden folglich auch von ihnen geprägt. Im wesentlichen sind das der technologische (Standardisierung, Schnittstellen, Innovationsdynamik), der ökonomische (Märkte), der soziokulturelle (Akzeptanz, Nutzungsverhalten) und der politisch-administrative Kontext. Die zahlreichen intersystemischen und personellen Verflechtungen in Steuerungsgremien bieten Ansatzpunkte für die politische Gestaltung großer technischer Systeme. Das Ausmaß, mit dem diese Kontexte bzw. Umwelten jeweils die Infrastruktur prägen, ist variabel und teilweise politisch steuerbar. Auch für technische Infrastrukturen gilt, was König generell für die öffentliche Verwaltung feststellt: »Es muss jeweils entschieden werden, welche Funktionen der Staat in seiner sozioökonomischen Umwelt wahrnimmt $\ll^{26}$.

Für die Politik kann das z. B. die Frage bedeuten, ob bei der Gestaltung einer technischen Infrastruktur das Management ausgetauscht oder die "Spielregeln" (Geschäftsordnungen, Definition von Universaldiensten) geändert werden müssen - oder beides. Weitere politische Entscheidungen im Zusammenhang mit der Gestaltung technischer Infrastrukturen sind die Gestaltung der Rechtsform (als $\mathrm{GmbH}$ oder als AG) und der Auflagen zur Erbringung bestimmter Leistungen (z. B. die Universaldienste). Die Privatisierungsdiskussionen über die Deutsche Bahn, die Telekom und viele kommunale Versorgungsnetze haben gezeigt, dass nahezu alle Einzelschritte politisch umstritten waren und immer sein werden:

26 K. König, »Gute Gouvernanz als Steuerungs- und Wertkonzept des modernen Verwaltungsstaates « in: W. Jann / K. König / Ch. Landfried / P. Wordelmann (Hg.), aaO. (FN 16), S. 227. 
- Ob überhaupt privatisiert werden soll,

- ob bei der Gründung einer - eventuell gemeinnützigen - GmbH die öffentliche Hand strategisch wichtige Funktionen in den jeweiligen Organen (Gesellschafterversammlung, Vorstand, Aufsichtsrat) behält,

- ob im Falle eines Börsengangs die Aktien breit gestreut, an bestimmte - auch internationale - Unternehmen vergeben werden oder in öffentlicher Hand bleiben,

- ob die Geschäftsführung und andere Leitungspositionen »politisch« oder nach Managementkriterien besetzt werden,

- ob und welche Behörden den Privatisierungsprozess durchführen und später die privatisierte Infrastruktur überwachen,

- ob die Privatisierung in einem Schritt oder in mehreren Teilschritten erfolgt,

- ob die Infrastruktur zentralisiert oder dezentralisiert wird,

- ob und wie die Kompetenz zwischen Bund und Ländern aufgeteilt wird.

Bei jeder Infrastruktur sind also mehrere Kombinationen denkbar, die jeweils unterschiedliche Folgen für staatliche Gestaltungsmöglichkeiten haben. Das Spektrum reicht vom Verkauf einer Infrastruktur an ein privates Unternehmen ohne jegliche Auflage und gesellschaftsrechtliche Beteiligung des Staates bis zur Privatisierung mit dem Bund als Mehrheitsaktionär, politischer Besetzung aller Leitungsfunktionen und »Begleitung « durch eine Regulierungsbehörde mit weitreichenden Kompetenzen etwa bei der Lizenzierung.

In der Regel dürfte der Staat in den Organen der privatisierten Infrastruktur vertreten sein und dadurch seine Interessen wahrnehmen. Über die Effizienz dieses Modells kann man zu Recht streiten. Weder hat die Mehrheit kommunaler Vertreter in den Aufsichtsräten der großen Energiekonzerne die Energiepolitik sozialverträglicher gestaltet noch hat die Beteiligung der Landesregierungen an den Multimedianetzen immer die demokratiefreundlichste Option gefördert. Hinzu kommt, dass »der Staat «(die Regierung) nicht mit einer Stimme spricht. Es ist ein Unterschied, ob z. B. das Wirtschaftsministerium die Rechts- und/oder Fachaufsicht über die energie- oder verkehrswirtschaftlichen Infrastrukturen, an denen die öffentliche Hand in irgendeiner Form beteiligt ist, ausübt oder das Umweltministerium. Gerade bei Koalitionsregierungen ist der regierungsinterne Koordinationsbedarf bei der Gestaltung technischer Infrastrukturen in den politisch sensiblen Bereichen Energie, Verkehr und Medien sehr hoch und birgt ein hohes Konfliktpotenzial. So standen sich in den 1990er Jahren etwa in der Frage nach dem Einstieg in die Stromerzeugung durch Braunkohle (»Garzweiler II«) in NRW das "grüne« Umweltministerium und das SPD-geführte Wirtschaftsministerium gegenüber. Wenn man bedenkt, dass jedes Ministerium immer auch der Anwalt seiner jeweiligen Klientel ist, stellt sich auch die Frage, ob eine Mehrheitsbeteiligung des Staates bei privatisierten Infrastruktursystemen im Sinne einer sozialverträglichen Option immer wünschenswert ist. Die Eigeninteressen staatlicher Behörden am Erhalt ihres Einflusses dürfen nicht unterschätzt werden. Schließlich bedeutet der Verlust eines Aufsichtsratsmandats auch einen Prestigeverlust für das Ministerium und eine Schwächung der von ihm vertretenen »Linie«. 
Es ist in der Technokratiedebatte der 1960er Jahre immer wieder vor einer Vereinnahmung des Staates und der Gesellschaft durch die Technik und ihre vermeintlich zwingende Logik gewarnt worden. Interessanterweise trafen sich in der Kritik an der Technik und ihren Folgen konservative ${ }^{27}$ mit ausgesprochen linken und später grünen Positionen ${ }^{28}$. Während jene einen starken Staat und die Bürger vor dem Verlust ihrer Freiheitsrechte bewahren wollten, forderten diese eine Demokratisierung durch erhebliche Ausweitung der Bürgerbeteiligung beim Bau technischer Großprojekte. Die Verteidiger der Technik hätten eigentlich an beiden Fronten argumentieren müssen. Sie haben jedoch nur gegen die vermeintlichen Technikverhinderer im linken Lager gekämpft, aber die Widersprüche im eigenen Lager übersehen, die jetzt bei der Frage der Gentechnik sichtbar werden.

Die Technokratiedebatte wurde in den 1970er Jahren durch eine regelrechte Steuerungseuphorie abgelöst. Inzwischen ist die Steuerungseuphorie der 1970er verflogen - sowohl bei den Politikern als auch bei den Steuerungstheoretikern. Spätestens seit den 1980er Jahren kennzeichnen Schlagworte wie Steuerungskrise oder Unregierbarkeit die Situation. Man kann der autopoietischen Systemtheorie zumindest insofern dankbar sein, als sie mitten in der Euphoriephase an die Grenzen intentionalen Handelns durch den Staat erinnerte - wenngleich unter falschen Prämissen: die Postulierung von Subsystemen, die statt miteinander zu kommunizieren nur »diplomatische Noten« austauschen ${ }^{29}$.

Die Grenzen staatlichen Handelns haben noch andere Gründe. Neben der bereits erwähnten Zunahme der Komplexität gesellschaftlicher Bereiche und der damit verbundenen Vielfalt möglicher Folgen ist das vor allem die Lage der öffentlichen Haushalte und die generelle Skepsis gegenüber staatlichen Planungen angesichts des Zusammenbruchs der Planwirtschaften. Letzteres hat den Legitimationsdruck für diejenigen erhöht, die eine erhöhte Planung und Regulierung fordern. Deregulierung, Selbststeuerung und Liberalisierung sind scheinbar zu unhinterfragbaren Dogmen geworden. Es ist erstaunlich, mit welcher Selbstverständlichkeit die Politik die Borniertheit einer bestimmten wirtschaftswissenschaftlichen Schule übernimmt, als hätte es nie eine Diskussion über soziale Folgen, ökologische Risiken, Kollektivgüter oder Marktversagen gegeben. Dies einzufordern ist auch eine politische Angelegenheit. Selbst die Positionen der Ordoliberalen waren vielfach weiter als die derzeitigen Leitbilder der kompromisslosen Privatisierung ${ }^{30}$.

Die Steuerungstheorie hat den finanzpolitischen Spielräumen des Staates zu wenig Aufmerksamkeit gewidmet. Was als Steuerungskrise des Rechts oder Politikversagen aussieht, ist im wesentlichen eine Finanzkrise, zumal die Haushaltskonsolidierung zu einem Ziel an sich geworden ist. Gesetze zur Errichtung von Infrastrukturen können nur dann ihre Wirkung entfalten, wenn sie mit entsprechenden

27 E. Forsthoff, aaO. (FN 1); H. Schelsky, aaO. (FN 1).

28 C. Koch / D. Senghaas (Hg.), Texte zur Technokratiediskussion, Frankfurt a. M. 1991.

29 K. von Beyme, "Steuerung und Selbstregelung. Zur Entwicklung zweier Paradigmen« in: Journal für Sozialforschung 1995, S. 197-217.

30 H.-H. Hartwich, aaO. (FN 15), S. 183. 
Haushaltstiteln ausgestattet sind. Ein Gesetz zur Errichtung einer Großforschungseinrichtung geht ins Leere, wenn nicht gleichzeitig die Mittel für ihre Errichtung und ihren langjährigen Betrieb gesichert sind. Die Finanzkrise aller öffentlichen Haushalte ist - umgekehrt - ja gerade einer der wichtigsten Gründe für den Verkauf staatlicher Infrastrukturen. Die Bindung öffentlicher Mittel in Infrastrukturen erscheint somit als Schritt in die falsche Richtung. So wird aus der finanzpolitischen Not eine steuerungspolitische Tugend: Durch den Verkauf z. B. städtischer Versorgungsnetze wird nicht nur frisches Geld in die Kassen gespült, sondern auch der zeitgemäßen Forderung nach Entstaatlichung Genüge getan. Es bleibt abzuwarten, ob dieser Weg von den Bürgern akzeptiert wird. Es sieht nach einigen Bürgerentscheiden nicht immer danach aus ${ }^{31}$.

\section{Entideologisierung und nener Pragmatismus}

Die Wahlerfolge von »New Labour« und die Suche aller Volksparteien nach der »Neuen Mitte zeigen, dass die Wähler weniger ideologische Positionen als vielmehr die erkennbare Lösung von Problemen honorieren. Ob die Arbeitslosigkeit mit einer nachfrage- oder angebotsorientierten Politik bekämpft wird - beides war jahrzehntelang ein Glaubenskrieg zwischen Keynesianern und Neoliberalen -, interessiert heute noch nicht einmal die verantwortlichen Politiker. Sie experimentieren statt dessen mit Modellversuchen jenseits gefestigter Dogmen. Es gilt die Ärzteweisheit: Wer heilt, hat recht ${ }^{32}$.

Auch die Steuerung technischer Infrastrukturen ist von diesem pragmatischen Denken geprägt. Es geht nicht mehr um die vermeintlich richtige Glaubenslehre als vielmehr um Effizienz. Auch für grüne und sozialdemokratische Politiker ist die Privatisierung zu einer Frage der Zweckmäßigkeit geworden. Allerdings ist die Politik immer wieder gefragt, wenn es um die Sicherung der Arbeitsplätze in staatlichen Infrastrukturen geht, die bei einer Privatisierung gefährdet sind. Auch nach einer Privatisierung bleibt der Staat dafür in der Gesamtverantwortung. Das kann zu Zielkonflikten insofern führen, als der Staat einerseits die Privatisierung fördert, aber auf der anderen Seite die Übernahme der politischen Verantwortung für die Folgen nicht ohne weiteres ablehnen kann. Sollte dies geschehen, droht die Privatisierungspolitik am Votum der Bürger zu scheitern. Es bleibt somit das Dilemma, für die Folgen der Privatisierung einstehen zu müssen, die man nicht verursacht hat.

31 Ein bemerkenswerter Indikator für ein mögliches Ende des Privatisierungsbooms ist auch die deutliche Mehrheit, die im Mai 2001 bei einem Bürgerentscheid gegen den Verkauf der Düsseldorfer Stadtwerke stimmte. Es sei dahingestellt, ob sich die Bürger mehr darüber ärgerten, dass der Bürgermeister das Geld schon für andere Projekte eingeplant hatte, oder man eine Verschlechterung der Dienstleistungen befürchtete. Eine wichtige technische Infrastruktur bleibt damit in öffentlicher Hand.

32 Selbst das ZK der kommunistischen Partei Chinas hat seine Position zur Marktwirtschaft durch die Doktrin ersetzt: »Es ist egal, ob die Katze weiß oder schwarz ist. Hauptsache, sie fängt Mäuse.« 
Der Hinweis auf die ausschließlich wirtschaftlichen Erwägungen des neuen Besitzers sind zwar korrekt, aber politisch unakzeptabel. So wird auch in Zukunft immer wieder nach dem Staat gerufen werden, wenn Züge zu spät kommen, Telefontarife zu hoch oder die Stromtarife intransparent sind. Vielleicht ist es aber nur ein Problem der politischen Kommunikation und Kultur, dass man gerade in Deutschland vom Staat fast alles, aber von der Bürgergesellschaft fast nichts erwartet und dass man die Zusammenhänge zwischen Privatisierungspolitik, Haushaltskonsolidierung und der Bereitstellung technischer Infrastrukturen nicht sieht.

\section{Resümee}

1. Technische Infrastrukturen und großtechnische Systeme erbringen für die Gesellschaft elementare Leistungen und bedürfen daher der staatlichen Gestaltung.

2. Der moderne Staat sieht sich einer wachsenden Anzahl gesellschaftlicher Akteure und Institutionen gegenüber, mit denen er seine Gestaltungskompetenz teilen muss. Die Folge davon sind immer mehr Netzwerke, in denen die Exekutive mit ihrer Klientel nicht nur über Ziele sondern auch über Verfahren verhandelt. Eine Alternative zum verhandelnden Staat gibt es nicht. Die Folge ist ein steigendes Demokratiedefizit.

3. Technische Infrastrukturen folgen nicht einer einzigen spezifischen Rationalität sondern sind in mehrere Kontexte (technologisch, soziokulturell, ökonomisch, politisch) eingebunden, deren jeweiliger Einfluss teilweise politisch steuerbar ist.

4. Die Besonderheit technischer Netzwerke als Steuerungsobjekt ergibt aus der technischen Innovationsdynamik, die sich trotz soziokultureller Einbindung nur beschränkt steuern lässt. Durch die laufenden Veränderungen des »Standes der Technik « entsteht ein permanenter Steuerungsbedarf.

5. Die Steuerungskrise des Staates ist wesentlich eine Finanzkrise. Ohne finanzielle Spielräume zielen Steuerungsversuche weitgehend ins Leere. Demgegenüber verbleibt immer noch die Möglichkeit, über die Gestaltung von Rahmenbedingungen Einfluss auf Infrastrukturen zu nehmen. Aus der finanzpolitischen Not wird eine steuerungstechnische Tugend: Entlastung des Staates durch Beteiligung privater Akteure.

6. Auch bei völliger Privatisierung technischer Infrastrukturen verbleibt beim Staat die politische Verantwortung für ihre jeweiligen Folgen und Risiken.

7. Staatliche Akteure müssen ihre Rolle als Moderatoren und Koordinatoren organisierter Interessen lernen, da sie nicht dem traditionellen Verwaltungshandeln entspricht. Dazu gehört ein stärkeres Gewicht auf informelle Verhandlungsverfahren zu Lasten des Verwaltungsrechts.

8. Ideologische Motive bei der Privatisierung treten hinter pragmatischen und finanzpolitischen zurück: Wer heilt, hat recht, und erlaubt ist, was Probleme löst und sich zudem rechnet. 


\section{Zusammenfassung}

Technische Infrastrukturen und großtechnische Systeme erbringen für die Gesellschaft elementare Leistungen und bedürfen daher der staatlichen Gestaltung und politischer Verantwortung. Ihre Besonderheit als Steuerungsobjekt ergibt sich aus der technischen Innovationsdynamik, die sich nur beschränkt steuern lässt. Die Steuerungskrise des Staates ist im wesentlichen auch eine Finanzkrise. Demgegenüber verbleibt immer noch die Möglichkeit, über die Gestaltung von Rahmenbedingungen Einfluss auf Infrastrukturen zu nehmen. Auch bei einer Privatisierung verbleibt beim Staat die politische Verantwortung für ihre jeweiligen Folgen und Risiken.

\section{Summary}

Technological infrastructures and larger technical systems have a great relevance for the society. This is the main reason why they should be shaped by the state. The state is also responsible for larger technical systems and their risks. The specific character of these systems as an object of shaping is the dynamic of innovations. The innovation process cannot be completely controlled by the state. The impossibility of shaping or even controlling technology is also the effect of the finance crisis of the state. There are other ways and strategies to gain the control over larger technical systems, for instance public-private-partnership. Even when these systems where completely in the hands of private companies the state still is responsible for the risks. 